\title{
Intraoperative ultrasound in robot-assisted partial nephrectomy: State of the art
}

\author{
Giacomo Di Cosmo, Enrica Verzotti, Tommaso Silvestri, Andrea Lissiani, Roberto Knez, Nicola Pavan, \\ Michele Rizzo, Carlo Trombetta, Giovanni Liguori \\ Università degli Studi Trieste, Urology Department - Cattinara Hospital, Strada di Fiume 447, Trieste, Italy.
}

\begin{abstract}
Summary Introduction: Nephron-sparing surgery (NSS) is of one of the most studied fields in urology due to the balancing between renal function preservation and oncological safety of the procedure. Aim of this short review is to report the state of the art of intra-operative ultrasound as an operative tool to improve localization of small renal masses partially or completely endophytic during robotassisted partial nephrectomy (RAPN).

Material and methods: We performed a literature review by electronic database on Pubmed about the use of intra-operative US in RAPN to evaluate the usefulness and the feasibility of this procedure.

Results: Several studies analyzed the use of different US probes during RAPN. Among them some focused on using contrastenhanced ultra sonography (CEUS) for improving the dynamic evaluation of microvascular structure allowing the reduction of ischemia time (IT). We reported that nowaday the use of intraoperative US during RAPN could be helpful to improve the preservation of renal tissue without compromising oncological safety. Moreover, during RAPN there is no need for assistant to hand the US probe increasing surgeon autonomy.

Conclusions: The use of a robotic ultrasound probe during partial nephrectomy allows the surgeon to optimize tumor identifcation with maximal autonomy, and to benefit from the precision and articulation of the robotic instrument during this key step of the partial nephrectomy procedure. Moreover US could be useful to reduce ischemia time (IT).

The advantages of nephron-sparing surgery over radical nephrectomy is well established with a pool of data providing strong evidence of oncological and survival equivalency. With the progressive growth of robot-assisted partial nephrectomy (RAPN) techniques, the use of several tools has been progressively developed to help the surgeon in the identification of masses and its vascular net. In this short review we tried to analyze the current use of intra-operative ultrasound as an operative tool to improve localization of small renal masses partially or completely endophytic during RAPN.
\end{abstract}

KEY WORDS: Robotic surgery; Nephron-sparing surgery; Intraoperative ultrasound; Contrast-enhanced ultrasonography .

Submitted 6 July 2018; Accepted 12 July 2018

\section{INTRODUCTION}

Renal cell carcinoma (RCC) is the seventh most common urological neoplasm with an incidence of approximatively 270.000 new cases diagnosed each year worldwide. The rising incidence of kidney cancer is related to the improv- ing of imaging techniques such as contrast-enhanced ultrasonography (CEUS), magnetic resonance imaging (MRI) and computed tomography (CT) that are capable to incidentally diagnose small renal masses (SRM). In past decades SRM have been treated by radical nephrectomy with increased risk of chronic kidney disease (1). For this reason there's been increasing interest in using nephron-sparing surgery (NSS) techniques and nowadays NSS is the standard of care for Tla renal masses and several retrospective series as well as one prospective randomized controlled trial (RCT) including patients with organ-confined RCC of limited size, low T-stage (pTla), have demonstrated a comparable cancer specific survival (CSS) for NSS versus radical nephrectomy (RN) $(2,3)$.

Several studies focused on the ischemia time as a predictor of renal function reduction. In this context, in recent years we have seen an increasing use of intraoperative ultrasound (IOUS) probes and contrast-enhanced ultrasonography (CEUS). The use of ultrasound in the intraoperative renal surgery is able to provide indications regarding the parenchyma and vascularization of the kidney. In this paper we describe the different operative approaches and we also performed a short review to focus on the actual application of intraoperative ultrasound in robot-assisted partial nephrectomy (RAPN).

\section{TECNIQUE}

Instrumentation and technical characteristics

The frequency normally used for laparoscopy or robotic ultrasound guidance is between 7.5 and $10 \mathrm{MhZ}$. Especially probes with 7.5 Mhz can surely provide excellent images for distances between 1 and 4 centimeters. In this context ultrasound is able to detect tumors up to 3$4 \mathrm{~mm}$ diameter (4). Probe may be linear (with multiple longitudinal transductors) or convex. Linear probe is generally more effective to scan organs with large flat surfaces as liver or spleen while convex probes are usually better for curved surfaces as kidney. To obtain better resolution is sometimes useful to irrigate the surface with saline solution (5).

During laparoscopic or robot-assisted procedure, both the assistant or the surgeon can control the laparoscopic ultrasound probe. In this second case the laparoscopic 
probe might require adjustment of probe positioning with also a robotic instrument to reduce probe slippage from tumor surface $(6,7)$. There's also the possibility of using ultrasound probes directly related to the robotic arm through a grooved ridge on its ventral aspect that fits the robotic grasping instrument allowing control by the surgeon himself.

In different series of laparoscopic or robot-assisted partial nephrectomy (LAPN and RAPN) with laparoscopic ultrasonography, it has been described some difficulty during the identification of tumor borders because the transducer may be not perpendicular to the surface of the kidney. The robotic ultrasound probe, instead, can be handled independently by the surgeon, achieving difficult angles while maintaining perpendicular contact of the probe with kidney surface (7).

\section{Intraoperative CEUS}

CEUS plays a key role in the characterization of malignant renal lesions (8). RAPN may be carried out by clamping of hilar vessels or by selective clamping of tumor vessels that aloud to reduce ischemia effects on the whole kidney. Several techniques have been described to perform partial ischemia by identifying tumor vessels and clamping or ligating those who feed the tumor (9).

Finally the intraoperative ultrasonography (IOUS) can be used for surgery of renal neoplasms, associated with the presence of venous thrombus. In case the thrombus extension is not visible or palpable, ultrasounds may be essential to identify the distal portion of the thrombus itself (10).

The contrast agent used during the CEUS procedure enhances the kidneys for about 2 minutes in real-time post-injection of contrast. In case of chronic kidney disease renal parenchyma could be enhanced for a shorter period and with lower intensity (11). A second-generation contrast agent, SonoVue (Bracco, Milan, Italy) is widely used for the CEUS procedure. Each milliliter of this contrast agent contains stabilized microbubbles of sulfur hexachloride gas (12). The recommended dose for renal imaging using a single intravenous injection of SonoVue is 1-2.4 ml. The ultrasound contrast agent can actually be seen to flow into the renal parenchyma usually within 15-20 seconds after an intravenous injection of SonoVue. This contrast enhancement of the renal parenchyma starts with the medulla and spreads to the renal cortex as the kidney is perfused with ultrasound contrast agent. Intravenous aliquots of SonoVue may be repeated as necessary and most importantly, this contrast agent is not nephrotoxic, as it is excreted by the lungs. Thus, it can be used safely in patients with compromised renal functions

The intra-operative CEUS technique uses two images: a conventional B-mode or 2D mode (brightness mode) image of the tissue using low acoustic power that produces a two-dimensional image on the screen and a contrast-enhanced mode (a contrast-specific) image which displays the reflection made by the spatial distribution of bubbles (Figure 1).

When an ultrasound wave falls on the microbubbles, they expand to almost double their original size and con-
Figure 1.

Intra-operative contrast-enhanced ultrasonography (CEUS) technique: conventional B-mode or 2D mode (brightness mode) image and contrast-enhanced mode image which displays the reflection made by the spatial distribution of bubbles.

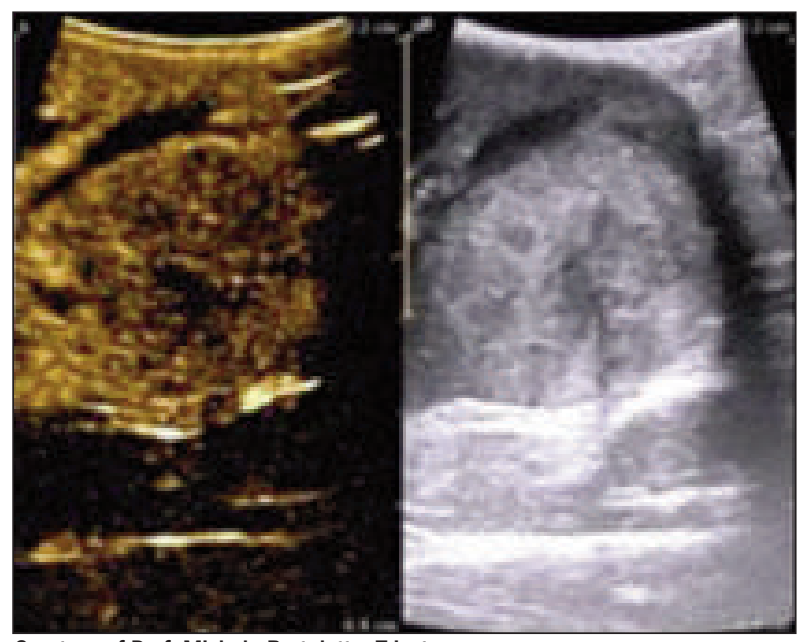

Courtesy of Prof. Michele Bertolotto, Trieste.

tract simultaneously, producing an oscillatory movement. This movement further results in the transmission of return signals to the US machine transducer (13), resulting in successful enhancement of the renal microvasculature and accurate tumor marking. A technique, which we are still developing, is sequential occlusion angiography. In this technique we capitalize on the ability to rapidly destroy or "rupture" the SonoVue microbubbles by increasing the ultrasound scanning frequency. This effectively clears the renal parenchyma or tissue being scanned of microbubbles and allows a second or subsequent intravenous injection of SonoVue to be administered immediately. In our hands, this is the real advantage of CEUS, which undoubtedly, seems to offer a better intra-operative imaging in comparison to power Doppler and Firefly.

The combination of CEUS and microbubble contrast agents allows a definite enhancement of contrast resolution, and inhibition of signals from stationary tissues. Although, SonoVue is more widely used for CEUS in most countries except the United States, there are a number of other alternative contrast agents available for this purpose.

\section{Technical procedures and our experience}

In our center we perform RAPN or LAPN for tumors sized $<4 \mathrm{~cm}$ with average PADUA score of 7 , most of times at lower pole of the kidney. Most of times, transperitoneal approach is used. Pre-operative staging is completed by chest and abdomen computed tomography (CT) to assess tumor morphology and vascular anatomy of the hilum. After identification and exposure of hilum, the surgeon release the Gerota's fascia (in case of posterior mass and transperitoneal approach, full mobilization of kidney is required). After giving patient $12.5 \mathrm{mg}$ mannitol, warm ischemia is induced by bulldog clamping on the principal arteria. In this moment intraoperative ultrasound helps to localize the mass, the depth of penetra- 
tion inside the parenchyma and its relationship with the collecting system. Moreover, IOUS helps in delineation of tumor margins and peritumoral vascularization: especially a contrast-enhanced ultrasound can enhance the visualization of the tumor and its vascularization during RAPN or LAPN with more precision consequently increasing the diagnostic accuracy of the surgeon and aloud selective clamping.

\section{Review of studies}

We performed as well a comprehensive literature search by electronic bibliographic databases in PubMed up to March 2018 using the following keywords: "contrast enhanced ultrasound", "intra-operative ultrasound", "nephron-sparing surgery", "partial nephrectomy" and "robotic-assisted partial nephrectomy". The list of all electronically identified bibliographies and articles was then reviewed to distinguish potentially relevant studies including experiments, case reports, and reviews and preliminary clinical studies. We selected studies in the field of intra-operative ultrasound in laparoscopic and RAPN.

\section{Discussion}

RAPN is performed with different techniques based on surgeon preferences, tumor characteristics, patient factors and available technology. According to AUA and EAU guidelines partial nephrectomy should be offered to all patients with organ confined disease, with mass equal or lass than. Although several nephrometric scoring system have been developed $(14,15)$ to help the surgeon in planning surgical best approach and CT or MRI clearly show the relationship between the lesion and renal sinus fat and pyelocaliceal system or involvement of renal vessels, IOUS can provide more detailed real-time guidance in the operating room for selected Tl lesions. Moreover, IOUS helps determine whether the distance between a main or segmental blood vessel and the tumor is greater $3-5 \mathrm{~mm}(16,17)$. Therefore, there is a perceived need for IOUS especially if the tumor is intraparenchymal and complex according to the nephrometry score. First reports of IOUS to help identify renal cell carcinoma in patients with poorly visualized and non-palpable disease have been described in 1988 (18). IOUS could also reduce operative time and ischemia time because it increase mass delimitation in those cases with particularly dense perirenal adipose tissue with highly represented fibrous components. Assimos et al. reported using of intraoperative ultrasonography for tumor identification to obtain negative surgical margins during partial nephrectomy (19). Great attention has also been given to IOUS use in the identification of extrarenal venous extension, multifocality and associated renal cysts and has also been reported that the use of intraoperative ultrasonography influenced the choice of surgical approach in $13 \%$ of cases $(20,21)$.

In recent years, there has been a progressive shift from laparoscopic partial nephrectomy and RAPN due to the ability to reduce the warm ischaemia time (WIT) and learning curve during nephron-sparing surgery in robotic surgery (22). A prolonged WIT has been demonstrat- ed to be potentially dangerous for renal functions post partial nephrectomy, especially in patients with high risk factors, or underlying disorders such as hypertension, diabetes, and small vessel disease (23-25). Surgeons are motivated to avoid global ischemia and consequently reduce the WIT by ligating or clamping selective arteries that supply blood to the segment of the kidney containing the tumor helps achieve a lower WIT.

Intra-operative ultrasound seems to be highly useful for this purpose, as it can demonstrate real-time imaging of the renal vasculature. CEUS is capable of further reducing the WIT by aiding the process of selective clamping, since it permits real-time scanning of the macrovasculature and microvasculature of the kidneys without the need for removing the perinephric fat.

Kaczmarek et al. (7) performed RAPN using a robotic US probe for tumor identification in 22 patients. The Gerota's fascia was opened to expose the renal capsule around the tumor, hilar blood vessels were isolated and clamped in preparation for excision of the tumor under warm ischemia and renoraphy was performed by "sliding clip suture" technique (26). The ultrasound probe was introduced through the assistant port to help the recognition of the border between tumor pseudo-capsule and normal renal parenchyma. The location and extent of the tumor were visualized through the medium of real-time images, obtained from intra-operative ultrasound techniques. Images were produced and visualized by the surgeon using the TilePro feature of the da Vinci surgical system to produce a picture-on-picture image in the console screen to view the images. Finally In some studies have been described intra-operative ultrasound scan using a fourth robotic arm with ProART robotic drop-in probe $(6,27)$.

\section{Conclusions}

IOUS has been demonstrated as one of the most important tool to help surgeon in maximize the loss of nephrons and it can be performed both by using assistant port or robotic probe with surgeon directly handling the probe with sensible benefit from the precision and articulation of robotic instrument. Intraoperative CEUS can further reduce global WIT and thus may improve recovery of renal function. By facilitating selective arterial clamping during RPN and avoiding global ischemia it may decrease the risk of permanent loss of nephrons. Most importantly, CEUS can help us image the renal microvasculature, without affecting renal function. In addition, CEUS is capable of dynamic evaluation and quantification of microvasculature blood (capillary perfusion) in real time. When used in conjunction with a robotic ultrasound probe, CEUS can facilitate better visualization of renal vasculature and tumor and ultimately improving acumen and precision.

\section{REFERENCES}

1. Hollenbeck BK, Taub DA, Miller DC, et al. National utilization trends of partial nephrectomy for renal cell carcinoma: a case of underutilization? Urology. 2006; 67: 254-9. 
2. Gratzke C, et al. Quality of life and perioperative outcomes after retroperitoneoscopic radical nephrectomy $(R N)$, open $R N$ and nephron-sparing surgery in patients with renal cell carcinoma. BJU Int. 2009; 104:470.

3. Van Poppel $H$, et al. A prospective, randomised EORTC intergroup phase 3 study comparing the oncologic outcome of elective nephron-sparing surgery and radical nephrectomy for low-stage renal cell carcinoma. Eur Urol. 2011; 59:543

4. Polascik TJMF. Intraoperative sonographic evaluation of the Kidney. AUA Update Series. 1997; 16:137.

5. Lirici, et al. Laparoscopy ultrasonography: limits and potential of present technologies. Endosc Surg Allied Technol. 1994; 2:127-133.

6. Rogers CG et al. Maximizing console surgeon independence during robot-assisted renal surgery by using the Fourth Arm and Tile Pro. J Endourol. 2009; 23:115-121.

7. Kaczmarek BF, et al Robotic ultrasound probe for tumor identification in robotic partial nephrectomy; initial series and outcomes. Int J Urol, 2013; 20:172-176,

8. Bertolotto $M$, et al. Renal masses as characterized by ultrasound contrast. Ultrasound Clin. 2013; 8:581-592.

9. Gill IS, Eisenberg MS, Aron M, et al. Zero ischemia partial nephrectomy: novel laparoscopic and robotic technique. Eur Urol. 2011; 59:128-134.

10. Hsu et al. Laparoscopic radical nephrectomy incorporating intraoperative ultrasonography for renal cell carcinoma with renal vein tumor thrombus. Urology 2003; 61:1246-1248.

11. Piscaglia F, et al. The EFMBSU Guidelines and Recommendations on the clinical practice of Contrast enhanced ultrasound (CEUS): update 2011 on non-hepatic applications. Ultraschall Med. 2012; 33:33-59.

12. Greis C. Technology overview: SonoVue (Bracco, Milan) Eur Radiol. 2004; 14(supp 8):11-15.

13. Uhlendorf V, et al Acoustic behavior of current ultrasound contrast agents. Ultrasonics. 2000; 38:81-86.

14. Kutikov, et al. The R.E.N.A.L. nephrometry score: a comprehensive standardized system for quantitating renal tumor size, location and depth. J Urol. 2009; 182:844-853.
15. Ficarra, et al. Preoperative aspects and dimensions used for an anatomical (PADUA) classification of renal tumours in patients who are candidates for nephron.sparing surgery. Eur Urol. 2009; 56:786-793

16. Li QL, et al. Significance of margin in nephron sparing surgery for renal cell carcinoma of $4 \mathrm{~cm}$ or less. Chine Med J (Engl). 2008; 121:1662-1665.

17. Lam, et al. Importance of surgical margin in nephron sparing surgery in the management of renal cell carcinoma. Nat. Clin Pract Urol. 2008; 5:308-317.

18. Gilbert BR, et al. Intraoperative sonography: application in renal cell carcinoma. J Urol. 1988; 139:582-284.

19. Assimos D, et al. Intraoperative renal ultrasonography: a useful adjunct to partial nephrectomy. J Urol 1991; 146:1218-1220,

20. Polascik TJ, et al. Intraoperative sonography for the evaluation and management of renal tumors: experience with 100 patients. J Urol 1995; 154:1676-1680.

21. Marshall, et al. Intraoperative sonography of renal tumor. J Urol 1992; 148:1393-1396.

22. Mottrie A, De Naeyer G, Schatteman P, et al. Impact of the learning curve on perioperative outcomes in patients who underwent robotic partial nephrectomy for parenchymal renal tumours" Eur Urol. 2010; 58:127-132.

23. Clark MA, et al. Chronic kidney disease before and after partial nephrectomy. J Urol. 2011; 185:43-48.

24. Thompson RH, et al. Every minute counts when the renal hilum is clamped during partial nephrectomy. Eur Urol. 2010; 58:340345 .

25. Campbell SC, et al. Guideline for management of the clinical T1 renal mass. J Urol, 2009; 182:1271-1279.

26. Benway BM, et al. Robotic partial nephrectomy with sliding-clip renorrhaphy: technique and outcomes. Eur Urol. 2009; 55:592-599.

27. Rao AR, Gray R, Mayer E, et al. Occlusion angiography using intraoperative contrast-enhanced ultrasound scan (CEUS): a novel technique demonstrating segmental renal blood supply to assist zero-ischaemia robot-assisted partial nephrectomy. Eur Urol. 2013; 63:913-9.

\section{Correspondence}

Giacomo Di Cosmo, MD (Corresponding Author)

giacomo.dicosmo@gmail.com

Enrica Verzotti, MD

enrica.verzotti@gmail.com

Tommaso Silvestri, MD

tommaso.silve@gmail.com

Andrea Lissiani, MD

a_lissiani@gotmail.com

Roberto Knez, MD

r.knez@libero.it

Nicola Pavan, MD

nicpavan@gmail.com

Michele Rizzo, MD

mik.rizzo@gmail.com

Carlo Trombetta, MD

trombcar@units.it

Giovanni Liguori, MD

gioliguori33@gmail.com

Università degli Studi Trieste, Urology Department - Cattinara Hospital,

Strada di Fiume 447, Trieste, Italy 